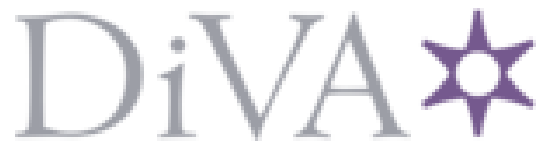

http://www.diva-portal.org

Preprint

This is the submitted version of a paper published in Language Dynamics and Change.

Citation for the original published paper (version of record):

Michaud, J. (2019)

Dynamic preferences and self-actuation of changes in language dynamics

Language Dynamics and Change, 9(1): 61-103

https://doi.org/10.1163/22105832-00901003

Access to the published version may require subscription.

N.B. When citing this work, cite the original published paper.

Permanent link to this version:

http://urn.kb.se/resolve?urn=urn:nbn:se:uu:diva-383951 


\title{
Dynamical preferences and self-actuation of changes in language dynamics
}

\begin{abstract}
A puzzling fact about linguistic norms is that they are mainly stable, but the conventional variant sometimes changes. These transitions seem to be mostly S-shaped and, therefore, directed. Previous models have suggested possible mechanisms to explain these directed changes, mainly based on a bias favoring the innovative variant. What is still debated is what is the mechanism that leads to such a bias. In this paper we propose a refined taxonomy of mechanisms of language change and identify a family a mechanisms explaining self-actuated language changes. We exemplify this type of mechanism with the preference-based selection mechanism that relies on agents having dynamical preferences for different variants of the linguistic norm. The key point is that if these preferences can align through social interactions, then new changes can be actuated. We present results of a multi-agent model and demonstrate that the model produces trajectories that are typical of language change.
\end{abstract}

\footnotetext{
Keywords

Language change - cultural evolution - actuation problem - preferences - utterance selection model - game theory
}

\section{Introduction}

Language is a social construct made of a large number of linguistic norms that are culturally transmitted and that form a semiotic system (Halliday. 1978) that is conventional inside a given speech community. An important question is to understand why and how established norms change. This question is not specific to linguistic norms and the discussion of this paper can be adapted to other social norms. Since language is a shared semiotic system, its dynamics can be influenced both by cognitive and linguistic factors, such as the ease of articulation or learnability, or by social factors, such as language contact or social structures. Since linguistic signs are mainly arbitrary (de Saussure, 1959), social factors can play a significant role in the evolution of a 
specific norm. In order to understand the dynamics of a language, it is important to understand the influence of each of these factors on the language dynamics. An important question asked by Blythe and Croft (2012) is: "How many qualitatively distinct possible mechanisms of language change are there?" (Blythe and Croft. 2012, p. 270). Based on the generalized theory of selection by Hull (1980, 2001, 2010) adapted to language evolution by Croft (2000); Baxter et al. (2006), Blythe and Croft (2012) proposed a classification of mechanisms influencing language change. They distinguished four categories of mechanisms, namely neutral evolution (NE), neutral interactor selection (NIS), weighted interactor selection (WIS) and replicator selection (RS). The RS mechanism groups all the mechanisms in which the variants are treated in a different manner by agents. Blythe and Croft (2012) argued that only RS can reliably account for S-shaped trajectories of change. In their model of RS, the innovative variant is given a selective advantage that causes its directed and S-shaped propagation through the population, but they did not provide an explanation for the origin of the shared advantage of the innovative variant and assumed it as given. It is unlikely that social factors and cognitive factors, which can both induce a selective advantage for a variant, influence the dynamics of a language in the same way and are, therefore, qualitatively different. The taxonomy proposed by Baxter et al. (2009) has to be refine to account for the factors influencing language dynamics.

With the help of a refined taxonomy of mechanisms of change, we investigate which type of selection mechanism can explain language change in absence of external (environmental) triggers to explain them. This is known as the actuation problem. The model we propose is an extension of the utterance selection model (USM) by Baxter et al. (2006), where the state of an agent not only considers the probability distribution of use of a variant of a linguistic variable (Labov, 2001; Tagliamonte, 2012), but also 
takes into account a preference vector associated with the different variants. The idea to add preferences in the state of the agents has been used by Acerbi et al. (2012) to explain fashion cycles. The influence of the preferences on the behavior of the agents is encoded in the updating rule of the agents rather than in the production rule as in Pierrehumbert et al. (2014). The idea originates from Michaud (2014), where a learning rule based on game theory and related to reinforcement learning (Sutton and Barto, 1998; Roth and Erev, 1995) has been proposed. We have adapted this updating rule to the USM and proposed an alignment mechanism based on a bias for social conformity (Asch, 1956). The proposed learning rule has a surprising connection to evolutionary game theory that we exploit to better understand the influence of preferences on the behavior of the agents. For instance, the learning rule obtained in our model can be written in a Replicator-Mutator (RM) equation, see for example Hofbauer (1985); Komarova (2004), and the preferences can be related to fitnesses of the RM equation. This model is able to simulate different selection mechanisms and provides some insights on what properties are needed for self-actuated changes to occur. The results of our model is compared with the models of Stadler et al. (2016) and Mitchener (2009).

The remainder of this paper is organised as follows. In section 2 , we review the main characteristics of time series of language change and discuss the available measures to quantify them. In particular, we discuss Kauhanen (2016) well-behavedness measures of language change and propose a few additional measures. In section 3 , we review Baxter et al. (2009); Blythe and Croft (2012) taxonomy of selection mechanisms of language change. We show that this taxonomy has to be refined, since the RS mechanism is shown to be covering qualitatively different selection mechanisms. We conclude this section by introducing the preference-based selection mechanism relying 
on the alignment of dynamical preferences for a given variant. In section 4 , we review the mathematical implementation of the USM and present our modification of it to include dynamical preferences. We also relate the revised learning rule to the RM dynamics. In section 5 , we present simulation results of our model and demonstrate that it successfully accounts for self-actuated changes that share all the characteristic properties of language change time series. We conclude this paper in section 6 , where we discuss the main results and compare them with other models.

\section{Properties of language change time series and how to measure them}

Measuring language change is a difficult task, since it implies measuring the usage of all the different variants of a sociolinguistics variable over a long period. The collection of such a data set would be really difficult, as a result, linguists rely on corpus studies to obtain a picture of the historical evolution of usage of the different variants. The resulting data typically takes the form of a time series of token frequency円 This is the data against which computational models should be validated. One of the main difficulty in characterizing time series of change is that no two changes follow the same trajectory, since they are independent realizations of a stochastic process.

In this section, we discuss the main characteristics of language change trajectories. Borrowing the terminology of Kauhanen (2016), we are interested in the well-behavedness of language change time series and in techniques to measure them and we propose a set of measures capturing the most important features of these trajectories.

\subsection{Characteristics of language change time series}

Language is a good example of a complex adaptive system (CAS) (Beckner et al., 2009. p.1-2) and its evolution is driven by interactions between speakers, since they change

\footnotetext{
${ }^{1}$ We are interested here in token frequency (frequency of occurrence of a given variant) rather than on type frequency (which is a measure of the number of available variants).
} 
their behavior in an adaptive manner determined by their interaction history. When a particular feature of the linguistic system changes from one variant to another (change in the proportion of speakers using the different variants), then one can say that a language change has occurred. Looking at the evolution of use of the different variants one can construct a time series of usage frequency of the different variants, which is the basis on which language change can be studied.

Since the linguistic system is mainly stable for communication efficiency (Lewis and Laland, 2012), structural and grammatical features of the linguistic system, such as word order, are not expected to change often (de Saussure, 1959; Labov, 2011) and most of the time, one variant (order) is dominating and most of the speakers use it. There are other properties of linguistic systems that are much more versatile and can change on a much shorter time scale. These properties tend not to be grammatical but lexical and their dynamics is closer to fashion cycles (Acerbi et al. 2012). An example of this kind of versatile linguistic trait are intensifiers (Partington, 1993; Tagliamonte, 2008; De Clerck and Colleman, 2013). Intensifiers such as VERY, PRETTY, so usually modify an adjective or an adverb, see also Ito and Tagliamonte (2003). It is relatively easy to introduce a new intensifier in such a way that the intensifying use is understood from the context. One possible source of new intensifiers are mass noun, an example is provided by massa's in Flemish (De Clerck and Colleman, 2013). The choice of an intensifier seems to be highly correlated with the linguistic community (De Clerck and Colleman, 2013; Tagliamonte, 2008) and it is reasonable to assume that social factors are dominant in their selection.

When discussing of language change, one assumes that we are changing from one variant to another, which implies that the change is, somehow, directed. A good discussion of the directedness of changes can be found in Stadler (2016), see also 
Haspelmath (2004); Newmeyer (1998). One of Stadler's conclusion is that directedness means that when a change is initiated, i.e. when a variant frequency of use starts to decrease in favor of another, the change continues until the invading variant becomes the new norm for the particular linguistic feature studied. The notion of directedness not only assumes an origin and an end, but also the monotonicity of the trajectory of change between the two.

If the language change time series are directed and monotonous, one can question the shape that this trajectories should have. According to Greenberg (1960); Bailey (1973); Kroch (1989); Denison (2003); Blythe and Croft (2012); Weinreich et al. (1968) language change time series are best approximated by an S-shaped curve similar to the logistic growth found in biological evolution. S-shaped curve trajectory is now established as a kind of template for change (Chambers et al. 2002).

From the previous discussion, we can conclude that a trajectory of change is wellbehaved if most of the time one variant is dominating and when a change occurs it is monotonous, directed and S-shaped. The frequency of change is expected to be higher for lexical features than for grammatical features.

\subsection{Origin and actuation of changes}

To be complete, a theory of language change should not only explain the shape of the time series, but also provide an explanation for the origin of changes and a mechanism to actuate them. Models of language change (Blythe and Croft, 2012; Pierrehumbert et al. 2014) usually assumed that the innovative variant has an advantage over the currently used variants, which leads to the replacement of the current variant by the innovative one, but do not specify where the advantage comes from. This is known as the actuation problem and has been stated by Weinreich et al. (1968) as:

Why do changes in a structural feature take place in a particular language at a given time, but not in other languages with the same feature, or in the same language at other 
times? This actuation problem can be regarded as the very heart of the matter. (Weinreich et al. 1968, p. 102)

An explanation for the change of some properties of the language is that a change in the environment led to the need of new concepts and new names. For example, the technological development creates a need for new terminology and any words filling these gaps will have an advantage over existing word referring to other things or concepts. So this is an external trigger of change. But changes in the environment are unlikely to explain structural changes or other changes in the fine structure of the linguistic system itself, such as the choice of intensifiers, which requires an internal trigger for the change.

A possible explanation for the internal actuation of a change has been provided Stadler (2016); Stadler et al. (2016). Using a momentum-based approach based on the concept of age vectors, Stadler (2016); Stadler et al. (2016) demonstrated that changes can be randomly actuated based on the momentum of variants, that is, the instantaneous trend of change of a given variant. In this scenario, speakers keep track of variation at two time scales and a change can be initiated whenever a significant amount of the population measures the same trend. This eventually occurs as a prediction-driven instability (Mitchener. 2009), which is an instability originating in the fact that the speakers who find a trend will follow it and amplify it. As a result, the trend becomes measurable by other agents who themselves start to follow, leading to a snowball effect or to an informational cascade (Pierrehumbert et al., 2014). Mitchener's model tends to create regular transitions that do not account for the relatively rare aspect of language change, in fact, it tends to create relatively regular changes from one variant to another. Stadler's model provides a satisfying explanation of the self-actuation of 
change, but assumes that whatever the new variant is, it has an advantage over the currently used variant.

The kind of self-actuated changes documented in Stadler et al. (2016); Mitchener (2009) also arises in the study of fashion cycles, since it is necessary for a new fashion to propagate through a population that it is innovated by some agents and copied by other agents. Acerbi et al. (2012) proposed a mathematical model relying on copying independently a cultural trait and the preference for that trait. They claim that the presence of preferences helped to understand the logic of fashion cycles. In their mechanism, a preference for a trait arises in the population (the origin of this preference is not stated) and then propagates through the population. Agents with the preference tend to copy the preferred trait more, leading to a propagation of the trait. Once agents possess both the preference and the trait, they tend to keep the trait and forget the preference, and, as a result, the trait starts to disappear from the population, closing the fashion cycle. Their mechanism assumes that the trait never reaches full consensus, otherwise, it would not decrease and stabilize as the new convention. In all these models, whenever an alignment of the bias or of the preference arises in the population the advantageous trait or variant propagates through the population. We can conclude that a shared bias for a variant leads to the directed propagation of this variant in the population. But as we mentioned in 2.1, fashion-like changes are a good description of lexical changes, but not of structural changes and this kind of explanation fails to provide a satisfying answer to the actuation problem. In order to gain insights in the actuation problem, we develop a theory of language change able to explain both structural changes and lexical changes in a single framework and to account for both external and internal triggers of change. 


\subsection{Quantifying the change}

Kauhanen (2016) proposed to measure the well-behavedness of a language change time series by using three measures: a dominance measure, a shifting measure and a monotonicity measure. He then grouped them together to form a well-behavedness measure. We describe them below.

The dominance measure is related to the fact that language is mainly stable and changes relatively rarely. A variant is said to be dominant at a given time if it is used by more than a given threshold the population. For example, a variant is $95 \%$ dominant if more than $95 \%$ of the population use it. The dominance score is computed by measuring the fraction of time that one variant is dominant and can be computed for different thresholds.

The monotonicity measure is related to the directedness of the changes (Stadler et al. 2016; Stadler. 2016), since a change that is monotonic is also directed. Kauhanen's monotonicity measure is computed over to complete trajectory and fluctuations occurring when one variant is dominant significantly contribute to that measure, that is, the monotonicity measure not only measures the monotonicity during changes, but also between changes. This measure might be difficult to interpret properly and is quite sensitive to the resolution of the time series. This is expected, since at a fast time scale a lot of variation can be expected, whereas for a lower time resolution, the variability of speakers averages out and the trajectory becomes more regular.

The third measure introduced by Kauhanen is the shifting measure. It is a measure of the number of shifts in the trajectory. The shifts are defined as the period between the change of dominance from one variant to another one. This measure can be normalized to a density of shifts to make it independent of the length of the time span 
during which the time series is recorded. This measure can also be used to estimate the frequency (or rate) of change, see, for example, Stadler et al. (2016).

While Kauhanen's measures capture the main properties of language change trajectories, they can be complemented by other measures to obtain a finer quantification of the trajectories. Therefore, we propose three additional measures: a measure of smoothness, a measure of the length of shifts and a measure of logisticness. The smoothness measure complements the monotonicity measure and is less sensitive to local fluctuations. The measure of the length of shifts complements the shifting measure by not only measuring the number of shifts, but also their length. Finally, the logisticness measure provides a measure of the shape of the time series, which is not accounted for in Kauhanen's measures.

The smoothness measure is obtained by looking at the time series at regular intervals and by measuring the overall tendency-to-change direction. For a given length of the interval $l$, the measure computes the density of change of direction when considering triplet $(t, t+l, t+2 l)$. If the value of the time series at $t+l$ is an extremum over the triplet, then there is a change of direction. This measure is not perfect and finer measure should be developed to account for the monotonicity and smoothness of the time series in a better way.

The measure of the length of shifts is computed by averaging the length of the different shifts occurring in a time series. Since they are dependent of the dominance level used to compute them, the length of shifts measure is also dependent on this parameter.

In order to characterize the shape of the transition, we introduce a measure of $S$ shapedness. Since there are many different functional forms for a S-shape curve, see Ghanbarnejad et al. (2014), we choose to use the most widely used S-shape curve, 
namely the logistic function. We measure the logisticness by fitting the trajectories in the shifting intervals to a logistic function. The quality of the fit is given by the determination coefficient $R^{2}$. From this measure, we obtain a logisticness measure by averaging the determination coefficient of all the shifting trajectories. The resulting measure is a number between 0 and 1 . We assign the value 0 to the logisticness measure in case the time series does not have any shifts.

Below we summarise the 6 measures that we use to quantify the results of our model.

Dominance: the dominance measure the proportion of time one variant dominates the time series. For the model in this paper, we measured the 95\%-dominance.

Monotonicity: the monotonicity measures is based on the number of changes in an fixed sized interval. We choose a interval length of 20. Kauhanen's monotonicity measure depends on a parameter $\alpha$, which has been set as $\alpha=0.5$, similarly to what have been done by Kauhanen (2016).

Smoothness: the smoothness measure is similar to Kauhanen's monotonicity measure, but instead of accumulating data in an interval, it only considers to boundary values of the interval. For this measure, we used an interval length of size $l=10$, which corresponds to considering only the boundary points and the central point of the monotonicity measure. Therefore, we expect this measure to be correlated with Kauhanen's monotonicity measure.

Number of shifts: a shift is defined as the period between a change in dominance, i.e. the time interval between one variant stopping to be dominant and a new one becoming dominant.

Length of shifts: the length of shifts measures the average length of shifts when they occur and output 0 in the absence of shifts. 
Logisticness: the logisticness measure is obtained by fitting the trajectory from the shifting intervals to a logistic function and the logisticness is given by the averaged determination coefficient $R^{2}$.

These 6 measures captures all the main properties of language change time series. The frequency of changes is captured by the dominance and the number of shifts measures; the directedness of the changes is captured by the monotonicity, the smoothness and the logisticness measures; the shape of the transitions is captured by the logisticness measure and if more than one change occurred, then it means that new changes can be actuated.

\section{Taxonomy of language change mechanisms}

In the previous section, we have discussed the properties of language change times series and how to measure them. We now turn our attention to the mechanisms responsible for these changes. In this paper, we adopt a variationist approach to sociolinguistics (Labov, 2011; Tagliamonte, 2012). According to this approach the linguistic behavior of a speaker is variable and influenced by the patterns of variation of linguistic behavior to which she is exposed (Baxter and Croft, 2016). Therefore, the variationist approach is an example of usage-based model of language dynamics (Bybee, 2001, 2007, 2010). More specifically, we base our discussion on the linguistic theory of Croft (2000, 2008), which is a particular instantiation of a variationist and usage-based approach. This theory is based on the generalized theory of selection of Hull (1980, 2001, 2010).

We start by recalling Croft (2000) utterance selection model and clarify the terminology implied by Hull's generalized theory of selection. We then introduce the taxonomy of mechanism of language change proposed by Baxter et al. (2009); Blythe and Croft 
(2012) and discuss its limitation. In particular, we discuss the origin of the hypothesized selection advantage of incoming variants and show the need to refine the taxonomy of Blythe and Croft (2012). We conclude this section by presenting the preference-based selection mechanism of language change. This mechanism is able to explain the emergence of a selective advantage of a variant and the self-actuation of language change without the need for a functional bias or for an external trigger.

\subsection{Utterance selection model}

The generalized theory of selection designed by Hull (1980, 2001, 2010) considers the evolution of a replicator. The replicator comes in different variants and Hull's selection theory formalizes the process influencing the selection of a particular variant. The replicators can interact directly or indirectly through a medium, called the interactor, through which the interaction between replicators occurs. In Hull's theory, the variants of the replicators are replicated by the interactors, which leads to the selection (or absence thereof) of a particular variant of the replicator. These variants might be replicated differently by a given interactor either due to randomness in the replication process (altered replication) or because there is an advantage for the interactor to use a given variant more than the others (differential replication). Altered replication and differential replication can both lead to the selection of a variant. The selection mechanism is said to be replicator neutral if it is symmetric with respect to the variants, i.e. all the variants have the same probability to be selected.

Croft (2000, 2008) instantiated Hull's generalized theory of selection for language evolution. In this particular case, the replicator can be assimilated to the sociolinguistic variable (Labov, 2001, 2011; Tagliamonte, 2012) and can represent any properties of the linguistic system, which are called linguemes by Croft. The interactors are the speakers of the language and they replicate sociolinguistic variables by producing utterances 
that are exchanged between interactors, leading to the selection of some variant of the sociolinguistic variable. Since the selection occurs through the utterance production, Croft (2000, 2008) called this theory the utterance selection model (USM).

Using the USM to model language change has many advantages. The implementation of the USM by Baxter et al. (2006); Blythe and Croft (2012), detailed in Section 4.1, can be used to model many different language change mechanisms and has been used to test Trudgill's theory of new-dialect formation on the emergence of the New Zealand English dialect, see Baxter et al. (2009) and to model the apparent time construct used in sociolinguistics and the origin of the adolescent peak (Baxter and Croft. 2016).

This model is one of the few that can describe the formation and the subsequent change of conventions. Among other models that are able to model both the emergence and change of convention, the model of Mitchener (2009) is a population-based model that uses an age-structured population to explain change. The USM is simpler in that it does not require the population to be structured in order to trigger a change. The idea to use an age-structured population has also been used by Stadler et al. (2016), who uses a momentum-based approach to model language change and in particular, to account for their directedness.

From an analytic point of view, Baxter et al. (2006) and Michaud (2017) fully analyzed the model and obtained continuous time limits both at the agent level and at the population level. These analytic results can help to draw conclusions on the dynamics of the model and to understand the influence of a particular mechanism on the evolution of the system. In this paper, we do not go into the details of these fairly technical studies, but we will refer to specific results when needed. 


\subsection{Blythe \& Croft taxonomy}

An important question asked by Blythe and Croft (2012) is: "How many qualitatively distinct possible mechanisms of language change are there?" (Blythe and Croft, 2012, p. 270). Their answer was that there are four qualitatively distinct mechanisms of language change. They called these mechanisms replicator selection (RS), neutral interactor selection (NIS), weighted interactor selection (WIS) and neutral evolution (NE) and argue that NE and RS can also be found in biological evolution, whereas NIS and WIS are specific to cultural evolution. In a cultural evolutionary system, selection can be active either on the replicators or on the interactors.

The RS mechanism refers to any evolutionary advantage of a particular variant that improves its fitness in the communication process and selection is active on the replicators. This leads to a differential replication of the variants, i.e. the variants which have an evolutionary advantage are replicated more. As a result, one variant propagates through the population while the others fall out of use. The origin or this selective advantage is not clearly specified by Blythe and Croft (2012), but they suggest that the differential weighting between variants should be construed as a social valuation of variants by speakers, see for example the socio-historical model of Labov (2001). Baxter et al. (2009); Blythe and Croft (2012) distinguished two types of replicator selection: NIS and WIS. NIS refers to an altered replication of a given variant due to an asymmetry in the rate of interaction between speakers, even if there is no asymmetry of variant production by the speakers. NIS models the influence of the social structure and, in particular, of the social network underlying the interactions in a linguistic community (e.g., Milroy (1980) although RS is also part of this theory). NIS is symmetric with respect to interactors, because it only depends on the frequency of interactions between two speakers and affects them in a symmetric manner. 
WIS is similar to NIS, but accounts for an asymmetry in the interaction. When two speakers interact, there is an asymmetry in the way the utterances are treated and one speaker accommodates more to her interlocutor than the other way around (Giles. 1973; Giles and Powesland, 1997; Giles and Smith, 1979). WIS can, for example, account for leader-follower or adopter theories of diffusion of innovations (Rogers 2010; Labov, 2001; Milroy and Milroy, 1985; Nevalainen et al., 2011).

Finally, language change can happen completely randomly; when all the variants are given the same valuation and when there is no interactor selection. This only occurs in well-mixed populations or if the variability of the speakers has a greater influence of the time series than the other selection pressures. One property of NE is that a frequent variant is more likely to be adopted in a population than a less frequent one. This mechanism has been argued to be a plausible model of the frequency effects documented in usage-based theories of language change (Bybee, 2001, 2007, 2010).

\subsection{Where does differential replication comes from?}

In Blythe and Croft (2012), RS is modeled by introducing a population-wide asymmetry between the different variants, favoring one over the other. This kind of objective advantage of one variant really questions the origin of such an asymmetry. How did all the speakers agreed that one variant is better than another? A simple explanation would be a kind of functional bias, which gives that variant an advantage in communication. This is at odds with a social valuation explanation, since a social value should be agreed upon. In this section, we discuss the possible origins of selective advantage of variants of a replicator.

There are multiple factors influencing the dynamics of a language. These factors can roughly be categorized into three classes: cognitive, linguistic or socio-cultural. These factors can all influence the differential replication of a particular variant. In the 
literature, these factors have called by various names: Nedergaard Thomsen (2006) studied the different pressures acting on the variants and MacWhinney et al. (2014) refer to motivations of using one specific variant. These pressures/biases/motivations to use one variant more than the others constitute a individual valuation of the variants of the replicator and, according to the socio-historical of Labov (2001), these individual valuations of variants should align to lead to a selective advantage.

We now review some of the main factors that can trigger an advantage of a given variant. If the advantage of a variant is the same for all interactors one could call it objective. An objective advantage can originated in various biases, which can be cognitive, such as Fedzechkina (2014); Fedzechkina et al. (2016) preferences for variants that are easier to process, or they can be linguistic. Various linguistic biases such as: a regularization bias (Reali and Griffiths, 2009), a systematicity bias (Smith et al., 2010), a naturalness bias (Finley and Badecker. 2007), an expressivity bias (Kirby et al. 2015), etc. have been proposed. These biases are objective in the sense that the linguistic system is the same for all speakers and their judgment of regularity, systematicity, naturalness, expressivity, etc. are similar. This type of biases are trivially aligned in the population. Contrarily to cognitive and linguistic factors, socio-cultural factors, such as social conformity, see, for example, Asch (1956), are mainly subjective. The same variant can be socially valuated differently depending on the speech community it is used in. This means that such an effect should be attached to the speaker rather than to the community and a variant can propagate if there is an alignment of the individual valuations of this variant in the speech community. This type of subjective influences are of similar nature to the factors influencing trends and fashions formation and are qualitatively different from a cognitive or linguistic bias. In the literature, notions such as variant prestige (Le Page and Tabouret-Keller, 1985; Cedergren, 1987;; Tagliamonte, 
2015) have been developed to account for this type of influences. It is the requirement for an alignment of the individual subjective valuations, which makes subjective and objective biases qualitatively different.

As we have seen, there is a qualitative difference between objective and subjective factors influencing the individual valuation of variants by the speakers. This implies that the taxonomy presented in 3.2 has to be refined, since the RS mechanism accounts for qualitatively different mechanisms.

\subsection{Refinement of the taxonomy}

As we have seen in the previous section, the taxonomy of Blythe and Croft (2012) has to be refined. This conclusion was also reached by Mühlenbernd and Michaud (2017) by weakening the assumption that the selective advantage was objective, i.e. shared by all the population. They find out that if the bias is subjective and randomly changing then there is no selection of a particular variant and the different variants coexist in the population.

The main distinction that has to be made between different RS mechanisms is whether the valuation of variants is the same for every agent (objective valuation) and depends on the social context and personal preferences of the interactors (subjective valuation). This distinction leads to two sub-categories of RS, an objective RS (ORS) and a subjective RS (SRS).

An additional distinction which is useful to make between RS mechanisms is related with the time scale of change of the valuation. All types of biases evolve with time, but they do not evolve on the same time scale. On the one hand, cognitive biases evolve on a biological time scale and barely change on the time scale of language change. As a result, they can be well-approximated as static biases. On the other hand, social biases are highly variable and evolve on a faster time scale and cannot 
be considered as static but should dynamically evolve during language evolution. Linguistic biases are somewhat intermediary and can be considered either as static or as dynamic depending on which trait we are looking at. For lexical change, structural biases stay roughly constant and can be considered as static. For grammatical changes the situation is different since a change in such a feature leads to a reorganization of the linguistic system itself, making these pressures dynamic.

With these two additional criteria, the RS mechanism proposed in Baxter et al. (2009) should be refined into four different sub-mechanisms: (i) static and objective replicator selection (SORS); (ii) dynamic and objective replicator selection (DORS); (iii) static and subjective replicator selection (SSRS) and (iv) dynamic and subjective replicator selection (DSRS). It is important to note that a DSRS mechanism can be replicator neutral, whereas SORS, DORS and SSRS mechanisms are usually not. The type of RS studied in Blythe and Croft (2012) is static and objective. This type of selection mechanism models functional biases which vary weakly with respect of the time scale of language change. A DORS mechanism models a situation where an objective bias changes in the same way for the complete population. Such a pressure can originate in an environmental change or any other external changes that can be observed by all speakers. This is one possible explanation of an external trigger to language change. If the valuation is subjective and dynamic, then each individual prefers one variant or another and, as we discussed, if these preferences align then selection can occur. This means that a SSRS mechanism cannot explain selection, since a static bias cannot align in any ways and, if it is aligned it cannot be differentiated from a SORS mechanism. As a result, objective selection mechanisms model functional pressures and environmental changes, whereas subjective selection mechanisms model socio-cultural pressures and is the only class capable of modeling self-actuated 
changes, i.e. changes not triggered by an external source. Therefore, we will focus on DSRS in the rest of this paper.

\subsection{Preference-based selection}

In this paper, we are looking for a replicator neutral mechanism of language change that can explain the main properties of language change, see section 2, and provide some insights into the actuation problem. For the changes to be internally triggered, we need a DSRS mechanism in which agents evaluate the different variants individually complemented by a dynamic evolution of these valuations that leads to their population-wide alignment. In this paper, we refer to individual valuation as preferences.

The preference-based selection mechanism proposed in this paper is a particular instance of DSRS. It assumes that the state of an agent is given by a probability distribution over the possible variants for a given trait and by a corresponding preference vector. This two levels description of the state of the agents is similar to the model by Acerbi et al. (2012) and similarly to the logic of fashion cycle, preferences evolve and align when speakers interact. In order to understand how the mechanism works, let us assume that at a given point in time a variant of the replicator is consensual in the population. Due to altered replication, new variants can spontaneously emerge in the speech of individuals. If these newly introduced variants can influence the preferences of the interactors (for example through social conformity), then an interactor can grow a preference for a newly produced variant and starts to use it more, in other words, the interactor replicates her preferred variant differentially. Depending on the level of altered replication, the preference can either be reduced again or might be reinforced through random fluctuations. If it is reinforced, then other interactors will start to grow a preference for the same variant and a social valuation, i.e. a population-wide 
preference, starts to emerge. As a result, the variant is differentially replicated and propagates through the population. Once the preferred variant has reached consensus, the preference stops to grow and starts to decrease due to altered replication. At some point, the preference for the variant is forgotten and a preference for a new variant can start to form. The functional form of the preference decay controls the period of stability of a given variant and acts as a constraint on when a new change can be actuated.

In this mechanism, the dynamics of the preferences shares a lot of properties with Stadler et al. (2016) momentum-based selection mechanism. For instance, both preferences and momentum align due to random fluctuations in the population (altered replication), the momentum is based on random time correlations in the usage of the variants, whereas preferences are based on random spatial correlations. Since the time correlation can be observed by all agents in the population, the momentum of a variant in a population tends to align, which leads to the differential replication of the variant. The same logic applies to preferences, since spatial correlations can also be measured by different agents. The effect of preferences and momentum on language use is similar in that it leads to differential replication of the favored variant. This differential replication increases both the temporal and spatial correlations of usage in the population, leading to the propagation of the variant. The main difference between our model and Stadler's model is that we do not need the concept of age vector or any structure in the population (Mitchener, 2009) to explain language change. In fact a bias for social conformity (Asch, 1956) is sufficient to actuate new changes. This type of bias has been used by Jansson et al. (2015) to model the evolution of creole languages. The overall dynamics of the preferences follows the same logic as that of fashion cycles (Acerbi et al., 2012). It starts with the joint emergence of a trait and its corresponding 
preference. The preference is then propagated through an prediction-driven instability (Mitchener. 2009) (because differential replication amplifies correlations of usage) and when its reaches a saturated state, the preference decreases until it becomes comparable with the preference for another variant. At this point a new change can be actuated.

Since preferences can be associated with an evolutionary advantage in Croft's theory (Croft. 2000, 2008), the study of the dynamics of the preferences can explain how a social valuation can emerge in a population. As we will see, the proposed mechanism is in agreement with Labov socio-historical model of language change (Labov, 2001) and the dynamics of the preferences is similar to that found in Acerbi et al. (2012), which relates our work to the study of fashion cycles.

\section{Modeling language change}

In this section, we present our implementation of the preference-based selection mechanism. Our model is based on the implementation of the USM by Baxter et al. (2006) in which the learning rule is modified to include preferences for variants in a similar way as what is done in Michaud (2014) and in Acerbi et al. (2012) and we call this model the USM with preferences (USMwP).

We start by presenting the mathematical definition of the USM and then discuss our modification of it to include preferences. We also underline the relation of the new learning rule with the RM dynamics, which provides useful insights into the effect of preferences. As we have seen, the preferences need to evolve and align for the preference-based selection mechanism to work. In our implementation, we assume that the preferences evolve according to a bias for social conformity Asch (1956). This is sufficient for the mechanism to work. 


\subsection{Utterance selection model implementation}

Based on the evolutionary linguistic theory of Croft 2000, 2008), Baxter et al. (2006) designed the utterance selection model (USM) of language change. The USM is a stochastic agent-based model in which the replicator is a single sociolinguistic variable that can be instantiated in $V$ variants. The interactors are the agents in the system and they interact by exchanging utterances. The USM models any situations of cultural evolution, where the variants associated with a cultural trait are independent and where the knowledge of the speakers includes the frequency of use of each variants. In this model, $N$ agents are represented as nodes of a static network and interact along the edges of this network. The edge between agent $i$ and agent $j$ is weighted by a factor $G^{(i j)}$ modeling the frequency of interactions between the two agents. Using the matrix $G$ one can model different types of interactor selection. During an interaction, speakers replicate the variants by probabilistically generating an utterance $\boldsymbol{u}$ of length $L$ from the frequency distribution $x$ over the $V$ variants. In this case, the component $x_{v}^{(i)}$ is the frequency with which agent $i$ uses variant $v$. The utterance $\boldsymbol{u}^{(j)}$ is then communicated to agent $i$, who updates her representation. We now describe in more details the utterance production and the learning rule of the USM.

The utterances $u$ are produced from the frequency distribution $x$ by sampling $L$ tokens. The sampling process can be modified by a matrix $M$ accounting either for production errors or for innovations. The general production formula reads

$$
\boldsymbol{u}=M \frac{1}{L} \operatorname{Multi}(L, \boldsymbol{x}), \quad \text { or } \quad \boldsymbol{u}=\frac{1}{L} \operatorname{Multi}(L, M \boldsymbol{x}),
$$

where $\operatorname{Multi}(L, \boldsymbol{x})$ refers to a multinomial sampling process of length $L$ and parameter $\boldsymbol{x}$. The multiplication by the matrix $M$ models a mis-production effect, that is, it models altered replication. The matrix $M$ can also be applied to the frequency distribution 
$\boldsymbol{x}$ in which case the interpretation is slightly different and rather accounts for an innovation process or any other intra-linguistic process acting before the production (sampling). In Croft's theory, this is closer to differential replication than to altered replication and the matrix $M$ can be used to model both altered and differential replication. Both orderings have been used (Baxter et al. 2006. 2009; Blythe and Croft, 2012; Michaud, 2017) and the influence of a specific order is very limited and not usually observable in numerical simulations (Michaud, 2017). The parameter $L$ controls the variability of speech; if $L$ is small, utterances are short and the distribution $\boldsymbol{x}$ is not accurately reproduced, inducing a large noise in the transmission; if $L$ is large, however, the sampled distribution is accurate and the noise is weak and vanishes in the limit $L \rightarrow \infty$.

The updating (or learning) rule is formed by the weighted average of a process of selfmonitoring $S$ and a process of accommodation $A$ and given in Equation (2) for agent $i$ interacting with agent $j$ :

$$
\boldsymbol{x}^{(i), n+1}=\boldsymbol{x}^{(i), n}+\lambda\left[\left(1-h^{(i j)}\right) S\left(\boldsymbol{x}^{(i), n}, f\left(\boldsymbol{u}^{(i), n}\right)\right)+h^{(i j)} A\left(\boldsymbol{x}^{(i), n}, f\left(\boldsymbol{u}^{(j), n}\right)\right)\right],
$$

where $n$ refers to the $n$th interaction. The process of self-monitoring aims at reducing the difference between the frequency distribution $\boldsymbol{x}^{(i)}$ and the perceived utterance $f\left(\boldsymbol{u}^{(i)}\right)$ of an agent $i$, and the accommodation process aims at reducing the difference between the frequency distribution $\boldsymbol{x}^{(i)}$ and the perceived utterance $f\left(\boldsymbol{u}^{(j)}\right)$ of the interlocutor $j$. The function $f$ has been introduced in Blythe and Croft (2012), who used it to account for various perception biases and to implement an objective advantage of a given variant in order to model SORS. Stadler et al. (2016) used this function $f$ to implement a momentum-based mechanism of change, which is an example of a DSRS mechanism. 
The processes $S$ and $A$ are weighted according to an attention parameter $h^{(i j)}$, which represents the degree of accommodation to the interlocutor's utterance. The attention parameter can be used to model WIS. In the original version of the USM, the selfmonitoring and accommodation processes have been defined as

$$
\left\{\begin{array}{l}
S\left(\boldsymbol{x}^{(i)}, f\left(\boldsymbol{u}^{(i)}\right)\right):=\boldsymbol{u}^{(i)}-\boldsymbol{x}^{(i)} \\
A\left(\boldsymbol{x}^{(i)}, f\left(\boldsymbol{u}^{(j)}\right)\right):=\boldsymbol{u}^{(j)}-\boldsymbol{x}^{(i)}
\end{array}\right.
$$

which simply accounts for probability matching between the empirical frequency of use $\boldsymbol{u}$ and the frequency distribution $\boldsymbol{x}$. The main change to this rule has been made by changing the empirical frequency of use using the function $f$.

The model is completed by a (usually small) parameter $\lambda$ modeling the rate of learning. In other words, $\lambda$ represents a speaker receptiveness to the utterance heard. If $\lambda$ is close to one, the agent will fully adapt to the most recent utterance heard, whereas is $\lambda$ is small, the behavior of an agent will barely change. As a result, the learning parameter $\lambda$ can be interpreted as a measure of how long a particular utterance is remembered, see for example (Baxter and Croft, 2016). The parameters of the USM and their interpretation are collected in Table 1 .

[Table 1 here]

\subsection{Utterance selection model with preferences}

In this section, we discuss our mathematical implementation of the USMwP. In order to introduce preferences into the USM, we need (i) to modify the state space of the agents to include preferences; (ii) to define the influence of preferences on the behavior of agents and (iii) to define the preferences dynamics. The first step is the easier one, since we only need to associate to each agent a preference vector $\pi$ of length $V$ (the number of variants) and whose components take values between 0 and 1 . We could have chosen value between -1 and 1 as in Acerbi et al. (2012), but such a definition 
would only complicates the resulting accommodation rule, since we base our work on the learning rule used in Michaud (2014). The second step will be developed in section 4.2.1. The proposed learning rule draws on the fact that any discrete probability distribution can be interpreted as a mixed strategy to play a game (Tadelis, 2013). For a preference-based accommodation rule, we will show that the payoff matrix associated with the game is related to preferences of agents. We will also show that the obtained accommodation rule can be related to a RM dynamics, which helps to understand the behavior of the model. The third step consists in defining a dynamics for the preferences will be described in section 4.4 . As discussed in section 3.5 , we consider a simple bias for social conformity (Asch, 1956) as the driving force for alignment of preferences.

In our implementation of the preference-based mechanism, we assume that the influence of preferences on the behavior of agents is encoded in the learning rule and, more specifically, in the accommodation function $A$ of Equation (2). Introducing preferences at this level allows agents to treat different variants in a non-equivalent way. Since this heterogeneity in the treatment of variants is different for every single agent, this is an instance of subjective differential replication.

The accommodation rule with preferences is a generalization of the model of Michaud (2014). In this model, the choice of a language is associated with the mixed strategy to play an abstract coordination game (Cooper, 1998) and the learning rule is based on lateral inhibition, that is, successful variants are reinforced and unsuccessful variants are penalized. This type of strategy has been used in naming games experiments (Beuls et al., 2012; Steels and Loetzsch, 2012; Steels, 2007). In Michaud (2014), the penalization parameter is formed by the product of a objective cost and a subjective bias. 
In our model, we define a penalization vector $\boldsymbol{\alpha}:=\mathbf{1}-\boldsymbol{\pi}$ from the preference vector, i.e. the variants which are preferred more are penalized less during the learning process.

\subsubsection{Accommodation rule for 2 variants}

In the case of two variants, the coordination game between two agents $i$ and $j$ can be written in normal form (Cooper. 1998) as

$$
\Gamma:=\begin{array}{l|cc} 
& v^{(j)}=1 & v^{(j)}=2 \\
\hline v^{(i)}=1 & \mathrm{~S}_{11} & \mathrm{~F}_{12} \\
v^{(i)}=2 & \mathrm{~F}_{21} & \mathrm{~S}_{22}
\end{array},
$$

where $\mathrm{S}$ stands for success and $\mathrm{F}$ for failure in the coordination. The game $\Gamma$ is an abstract game for which the payoff matrix will be specified later. For every play of the game, agent $i$ accommodates to agent $j$ using the learning rule

$$
x_{1}^{(i), n+1}=\left\{\begin{array}{l}
x_{1}^{(i), n} \cdot\left(1-\alpha_{1}^{(i), n}\right) \text { if the outcome is } F_{21} \text { or } S_{22}, \\
x_{1}^{(i), n}+\alpha_{2}^{(i), n} x_{2}^{(i), n} \text { if the outcome is } F_{12} \text { or } S_{11} .
\end{array},\right.
$$

that is, she penalizes the unsuccessful variant and reinforces the successful variant.

This is an implementation of a confirmation bias. This rule has been used by different scholars (Michaud, 2014; Steels, 2007; Steels and Loetzsch, 2012; Beuls et al., 2013). In our case, we have to modify the lateral inhibition rule, since we only have access to utterance vectors, instead of individual outcomes. The learning rule then needs to take into account the probabilistic outcome of the game. For a given utterance vector $\boldsymbol{u}^{(j)}$, the variant 1 of agent $i$ will be penalized whenever agent $j$ uses variant 2 , that is, with probability $u_{2}^{(j)}=1-u_{1}^{(j)}$ and will be reinforced with probability $u_{1}^{(j)}$. The learning rule we obtain takes the form

$$
x_{1}^{(i), n+1}=x_{1}^{(i), n}\left(1-\alpha_{1}^{(i), n}\right)\left(1-u_{1}^{(j), n}\right)+\left(x_{1}^{(i), n}+\alpha_{2}^{(i), n} x_{2}^{(i), n}\right) u_{1}^{(j), n},
$$

which can be interpreted as: penalize $x_{1}^{(i)}$ by multiplying it by $1-\alpha_{1}^{(i)}=\pi_{1}^{(i)}$, if agent $j$ did not use variant 1 (occurring with probability $1-u_{1}^{(j)}$ ); otherwise, add to $x_{1}^{(i)}$ the 
probability weights coming from the penalization of the other variants (in this case, only variant 2). We can rewrite Eq. (6) in term of the jump $\delta x_{1}^{(i), n}:=x_{1}^{(i), n+1}-x_{1}^{(i), n}$, we obtain (see Proposition 1 in Appendix A for details)

$$
\delta x_{1}^{(i), n}=u_{1}^{(j), n} \sum_{w=1}^{2} \alpha_{w}^{(i), n} x_{w}^{(i), n}-\alpha_{1}^{(i), n} x_{1}^{(i), n} .
$$

The learning rule (7) has the properties to ensure that $x_{1}^{(i), n} \in[0,1], \forall n$ and that the total probability is conserved, see Proposition 2 in Appendix A

\subsubsection{Generalization to $V$ variants and USMwP}

For $V$ variants, the game structure is more complicated, but the accommodation rule is easy to generalize. For instance, Equation 7 still applies (see Appendix A.2 and, in general, we have

$$
\delta x_{v}^{(i), n}=u_{v}^{(j), n} \sum_{w=1}^{V} \alpha_{w}^{(i), n} x_{w}^{(i), n}-\alpha_{v}^{(i), n} x_{v}^{(i), n},
$$

which can be written in vectorial form as

$$
\begin{aligned}
\delta \boldsymbol{x}^{(i), n} & =\boldsymbol{u}^{(j), n}\left(\boldsymbol{\alpha}^{(i), n} \cdot \boldsymbol{x}^{(i), n}\right)-\operatorname{diag}\left(\boldsymbol{\alpha}^{(i), n} \otimes \boldsymbol{x}^{(i), n}\right) \\
& =: A_{\mathrm{p}}\left(\boldsymbol{x}^{(i), n}, \boldsymbol{u}^{(j), n}, \boldsymbol{\alpha}^{(i), n}\right)
\end{aligned}
$$

where the new accommodation term $A_{\mathrm{p}}$ is defined. The p index indicates the presence of the preference mechanism.

The definition of the new accommodation rule (9) is compatible with the accommodation rule of the USM (3). In fact, if all the penalization parameters $\alpha_{v}^{(i)}=1$, then (9) boils down to (3). We can now define the USM with preferences (USMwP) from equation Eq. (1) and Eq. (9). The generalized learning rule is given by

$$
\delta \boldsymbol{x}^{(i), n}=\lambda\left[\left(1-h^{(i j)}\right) S\left(\boldsymbol{x}^{(i), n}, \boldsymbol{u}^{(i), n}\right)+h^{(i j)} A_{\mathrm{p}}\left(\boldsymbol{x}^{(i), n}, \boldsymbol{u}^{(j), n}, \boldsymbol{\alpha}^{(i), n}\right)\right],
$$


where the self-monitoring function $S$ is left unchanged.

In order to better understand what the modified accommodation implies on the dynamics, we show an interesting link with evolutionary game theory (EGT) in the next section.

\subsection{Modified accommodation and RM dynamics}

In order to gain more insights into the dynamics of the USMwP given in Equation 10), we focus here on the effect of the revised accommodation rule. To do so, we set $h=1, \lambda=\delta t$ and let $\delta t \rightarrow 0$. By this procedure we obtain a continuous time limit of the dynamics induced by the accommodation with preferences. We have

$$
\dot{\boldsymbol{x}}^{(i)}=A_{\mathrm{p}}\left(\boldsymbol{x}^{(i)}, \boldsymbol{u}^{(j)}, \boldsymbol{\alpha}^{(i)}\right)
$$

and for each variant the corresponding equation is given by

$$
\dot{x}_{v}^{(i)}=u_{v}^{(j)} \sum_{w} \alpha_{w}^{(i)} x_{w}^{(i)}-\alpha_{v}^{(i)} x_{v}^{(i)}
$$

This equation is reminiscent of the RM dynamics, whose general form is given by

$$
\dot{x}_{v}=\sum_{w} x_{w} f_{w}(\boldsymbol{x}) Q_{w v}-\phi(\boldsymbol{x}) x_{v}, \quad \phi(\boldsymbol{x})=\sum_{w} x_{w} f_{w}(\boldsymbol{x})
$$

where $f_{w}$ is the fitness of the $w$ variant and $Q_{w v}$ is the mutation rate from variant $w$ into variant $v$. The average fitness of the population is given by $\phi(\boldsymbol{x})$. In Appendix A we show that we can rewrite Equation (12) in the form of Equation (13) if we set:

$$
\begin{aligned}
f_{v}^{(i), n}(\boldsymbol{x}) & :=-\alpha_{v}^{(i), n}=\pi_{v}^{(i), n}-1, \\
Q_{w v}^{(i j), n} & :=x_{v}^{(i), n}-u_{v}^{(j), n}+\delta_{v w} .
\end{aligned}
$$

In other words, the fitness of a variant $v$ for an agent $i$ is controlled by her preference for the variant and mutations are driven by the difference between the agent perceived 
frequency of use $x_{v}^{(i)}$ and the empirical frequency of use of her interlocutor $u_{v}^{(j)}$. We note that these fitnesses and mutation matrices are dependent on the speaker identity and are, therefore, subjective. This interpretation suggests that the different variants form an ecosystem inside the head of a speaker and that this ecosystem evolves according to a RM dynamics. Preferred variants are favored, since they have a higher fitness, and are replicated more. The mutation process is mainly driven by contacts and by the innovation/error process $M$ of the production process, which controls the utterance production and, therefore, $\boldsymbol{u}$. As a result, we obtain a competition between differential replication, modeled through the preferences, and altered replication, modeled through the innovation/error matrix $M$.

The coordination game (4) we started with is an abstract game and the payoff structure is not specified. From the RM dynamics, we can infer the payoff structure of the game $\Gamma$ by using the fact that if the fitness of a variant $f_{v}^{(i)}\left(\boldsymbol{x}^{(i)}\right)=A^{(i)} \boldsymbol{x}^{(i)}$ is linear, then $A^{(i)}$ is the payoff matrix of the game, which is given by

$$
A^{(i), n}=-\left[\begin{array}{ccc}
\alpha_{1}^{(i), n} & \ldots & \alpha_{1}^{(i), n} \\
\vdots & \vdots & \vdots \\
\alpha_{V}^{(i), n} & \ldots & \alpha_{V}^{(i), n}
\end{array}\right]
$$

since $\sum_{w=1}^{V} x_{w}^{(i), n}=1$. The $n$ index in Equation (15) highlights the time dependence of the payoff matrix and in Equation (14) the fact that fitnesses and mutation rates also evolve.

The resulting game is a game with imperfect information, since speakers do not know the preferences of their interlocutors, with evolving payoffs. The learning rule of the USMwP controls the evolution of the strategies of speakers and we have not yet specified the dynamics of the payoffs, i.e. the dynamics of the preferences. 
If the preferences do not evolve, then we can use EGT to predict the outcome of a simulation in simple situations. We consider three different scenarios: (i) there is one variant which is preferred by all the agents; (ii) the preferences are the same for all variants and (iii) all the preferences are randomly assigned. In the first case, all speakers prefer the same variant, then it is rationally the best to use that variant categorically and a rapid consensus will emerge in the population. If the preferences are the same for all the variants, the payoff obtained by an agent is independent of the variant used and in this case, no differential replication is possible and we recover the classical USM without preferences (up to a small modification related to the weighting of the self-monitoring process and the accommodation process). For random preferences, every agent will optimize her average payoff. The simplest strategy is to always use her preferred variant, because the payoff matrix has constant rows, which implies that the payoff of an agent is independent of the behavior of her neighbors. As a result, in the case of random static preferences, it is best for every agent to categorically use their preferred variant. These three scenarios will be tested through numerical simulations in section 5.1

As we mentioned in section 3.5. for the preference-based mechanism to work, the preferences should align. In terms of game theory, the alignment process aligns the payoffs of the game in such a way that the best rational strategy is to categorically use

the globally preferred variant, leading to a consensus. In the next section, we introduce our implementation of the preferences dynamics.

\subsection{Preferences dynamics}

The last missing component of the implementation is the preferences' dynamics. As mentioned in section 3.5, we consider an updating rule for the preference vector $\pi$, or equivalently for the penalization vector $\alpha$, based on a bias for conformity. To 
implement such a bias locally, agents have to be aware of the averaged speech of their neighbors. Let $V_{i}$ be the set of neighbors of $i$ on the network underlying the USM. We defined the average utterance $\boldsymbol{U}^{(i)}$ of the neighbors of an agent $i$ by

$$
\boldsymbol{U}^{(i), n}:=\frac{1}{\left|V_{i}\right|} \sum_{j \in V_{i}} \boldsymbol{u}^{(j), n}
$$

where $\left|V_{i}\right|$ denotes the number of agents in $V_{i}$. We define the updating rule for the preference vector $\boldsymbol{\pi}^{(i)}$ by

$$
\boldsymbol{\pi}^{(i), n+1}=\boldsymbol{\pi}^{(i), n}+\mu\left(\boldsymbol{U}^{(i), n}-\boldsymbol{x}^{(i), n}\right),
$$

where $\mu$ is a positive parameter controlling the rate of change of the preferences. The preference change in the direction of the difference $\boldsymbol{U}^{(i), n}-\boldsymbol{x}^{(i), n}$, aiming to align the behavior of an agent with the average behavior of her neighborhood. Therefore, Equation (17) implements of a social conformity bias (Asch. 1956). The choice to compare the internal representation $\boldsymbol{x}^{(i)}$ with averaged utterance $\boldsymbol{U}^{i}$ is motivated by the fact that we want to update our knowledge based on perceived information and since we do not have access to the information contained inside the brain of other agents, we must rely on their production. The parameter $\mu$ is usually chosen to be small, since values that are too large might lead to preferences exceeding their permitted values (between 0 and 1). In this case, one might introduce a $\mathrm{min} / \mathrm{max}$ limiter to project the preferences back onto the allowed interval. According to the updating rule (17), the preferences of underused variants are increased and preferences of the overused variants are decreased. It is important to note that if one variant is conventional, then the corresponding preference can only decrease, since it is not possible to overuse a categorical variant. Due to altered replication, the preference for innovative or erroneous variants can stochastically build up. For instance, the updating rule (17) also 
accounts for a surprise effect. Since the preference for a conventional variant can only decrease, at some point its preference will become similar to another variant. At this point, a new trend can be initiated that might lead to a directed change. In section 5.1 . we numerically test this dynamics, which provides some insights into the actuation problem.

Before performing numerical simulations, we can further discuss the effect that preferences have on the dynamics. For instance, the preferences acts as a memory of which variant was successful in the past. This can easily be shown by recurrence from Equation (17) and we obtain

$$
\boldsymbol{\pi}^{(i), n}=\boldsymbol{\pi}^{(i), 0}+\mu \sum_{m=0}^{n-1}\left(\boldsymbol{U}^{(i), m}-\boldsymbol{x}^{(i), m}\right),
$$

where the sum is over all previous interactions. The preference vector stores the cumulative history of differences of usage between an agent intended frequency $\boldsymbol{x}$ and the average behavior of her neighbors $\boldsymbol{U}$. The parameter $\mu$ controls the rate at which the memory changes. It is important to note that each component of the sum regularly crosses 0 , which means that long term effects are forgotten. In other terms, the preference updating rule models a short term memory. The time span of the memory depends on the parameter $\mu$. For very small values of $\mu$, the time span of the memory is very long and the effect of preferences is very small. As a consequence, the dynamics is dominated by altered replication influencing production. For larger values of $\mu$, the time span of the memory is fairly long and preferences generate long lasting trends that lead to differential replication. If $\mu$ is too large, the time span of the memory shortens and trends tend to change faster, leading either to fast and frequent changes or to more noisy trajectories. These prediction will be tested using Kauhanen's measures in section 5.2 


\section{Simulation results}

In this section, we test the USMwP and apply the measures designed in Section 2.3 to validate the proposed mechanism of language change. We test our hypothesis on a regular network of $N=20$ agents of degree $k=3$. The choice of a regular network is motivated by the fact that in such a network NIS is not active if all edges are sampled equivalently, which will be the case in all the simulations performed in this paper. We also set $h^{(i j)}=h=0.5$, so that there is no WIS either. The selection mechanisms active in this set up are either NE or RS. The USM without preferences is an instance of NE and adding preferences leads to the activation of some sort of RS, depending on the dynamics and initial condition of the preferences. Another motivation to use a regular network is that analytic results for the USM are known both at the agent level and at the population level (Baxter et al. 2006; Michaud, 2017). The linguistic variable considered has $V=2$ variants and the length of utterances $L=2$. In the updating rule (10), $\lambda=0.1$.

As discussed in section 3.5 , the preference-based mechanism relies on the competition between altered and differential replication. In order to tell apart both types of replication, we encode the altered replication in the innovation/error matrix $M$, through a parameter $q=10^{-3}$, controlling the innovation/error rate. For $V=2$ variants, the matrix $M$ is given by

$$
M=\left[\begin{array}{cc}
1-q & q \\
q & 1-q
\end{array}\right] .
$$

The differential replication process is encoded by the preference mechanism and, more specifically, by the parameter $\mu$ controlling the updating rate of preferences and driving the alignment of speakers' preferences. 
In Michaud (2017) it is shown that for regular networks a mean field approach can be used to derive the analytic form of the stationary distribution. This stationary distribution is related to the emergence of consensus in the population and it is shown that a phase transition exists between a phase in which the stationary distribution is bell-shaped and a phase in which the stationary distribution is U-shaped. The critical value $q_{*}(r)$ of the innovation/error rate as a function of $r=\frac{\lambda}{L N}$ is given by

$$
q_{*}(r)=\frac{r}{1+2 r+\sqrt{1+4 r}} .
$$

As mentioned in section 4.2.2, the USM is a special case of the USMwP when $\pi_{v}=0$, or $\alpha_{v}=1$, for all agent and for all variants $v$. For constant preferences $\pi_{v}=\pi_{*}$, Equation 20) can be generalized to

$$
q_{*}\left(r^{\prime}\right)=\frac{r^{\prime}}{1+2 r^{\prime}+\sqrt{1+4 r^{\prime}}}, \quad r^{\prime}=r\left(1-\pi_{*}(1-h)\right) .
$$

With this additional result, we have all the elements for discussing simulation results. In section 5.1 we discuss individual trajectories of the USM with preferences for various conditions in order to underline the importance of the preference dynamics for the preference-based mechanism to be efficient. In section 5.2, we apply Kauhanen's measures and a few others to fully characterize the dynamics of the model.

\subsection{Importance of preference dynamics}

We consider four different scenarios: the three scenarios with non-evolving preferences discussed in section 4.3 and the scenario in which preferences are updated according to Equation (17) described in section 4.4 . What changes between the different scenarios are the initial preferences $\pi_{v}^{(i), 0}$ and the value of $\mu$ controlling the preferences dynamics.

The four scenarios we consider are the following: 
Neutral preferences all preferences are equal and static. In terms of parameters, we set $\pi_{v}^{(i), 0}=0.5$ and $\mu=0$.

Random preferences preferences are randomly assigned and static. In terms of parameters, we sample $\pi_{v}^{(i), 0}$ from a uniform distribution over the interval $[0,1]$ and $\mu=0$.

Objective preferences variant 1 is preferred by all the agents and the preferences are static. In terms of parameters, we set $\pi_{1}^{(i), 0}=0.55$ and $\pi_{2}^{(i), 0}=0.45$ and $\mu=0$.

Dynamical preferences preferences change according to a conformity bias. In terms of parameters, we set $\pi_{v}^{(i), 0}=0.5$ and $\mu=0.05$.

These four scenarios cover four different selection mechanisms. The neutral preferences scenario is an instance of NE; the random preferences scenario is an instance of SSRS; the objective preferences scenario is an instance of SORS and the dynamical preferences scenario is an instance of DSRSZ

\section{[Figure1 1 here]}

Figure 1 illustrates the typical time series corresponding to the four scenarios. In panel a, a typical time series of the neutral preferences scenario is displayed. As expected, the trajectory is not very smooth and performs a random walk in the complete domain. With the parameters used, we are close to the critical value for the mutation parameter $q$. For instance, in our case $q_{*} \approx 9.34 \cdot 10^{-4}$, which is very close to the chosen $q=10^{-3}$. In panel $\mathbf{b}$, a typical time series of the random preferences scenario is displayed. We observe that the time series quickly reaches a relatively stable state where one variant is used slightly more than the other. If we look closely at the averaged values of prefer-

\footnotetext{
${ }^{2}$ We do not simulate here a DORS mechanism that models external triggers for language change since we focus on self-actuated changes. Furthermore, the dynamics of change of objective preference is not clear. We could have simulated random changes as in Mühlenbernd and Michaud (2017) with similar results (not shown).
} 
ences, we see that the preference for the variant which is used more is slightly higher. In this scenario, we expect every agent to be selfish and to use their preferred variant in a categorical manner. The average behavior of the agents is, therefore, in agreement with our prediction. In panel c, a typical time series of the objective preferences scenario is displayed. In this case, all agents quickly converge to using the preferred variant categorically, which is in agreement with the discussion in section 4.3 None of these scenarios have all the required properties for a well-behaved time series of change. As a result, it seems that having a static bias cannot lead to well-behaved language changes. This has also been observed by Pierrehumbert et al. (2014); when the biases distribution is random, no informational cascade is generated, but when the biases distribution is aligned, then informational cascades occur.

In panel $\mathbf{d}$ of Figure 1 a typical time series of the dynamical preferences scenario is displayed. The trajectory is mainly dominated by one variant. In the beginning, agents treat the two variants in the same way, leading to a short confused period. After this period, one variant takes over. The trend then reverses and the other variant reaches a consensual level. Then we observe a slow decrease of the preference for the consensual variant until around $t=1700$. At this point, a new change could be actuated, but random fluctuations lead to the conservation of the consensual variant. Later on, around $t=3800$ the preferences of the two variants become equivalent again and this time a directed change is triggered that eventually completes, leading to the adoption of the other variant. The change that occurs around $t=4000$ follows a Sshaped curve. We can conclude that the changes are not frequent, self-actuated and S-shaped, fulfilling all the requirements for a well-behaved trajectory of change. The dynamics illustrated by this trajectory suggests that the preference-based mechanism leads to an alternation of periods of stability and periods of change. The preferences 
are stabilizing conventions through differential replication and the memory effect discussed in section 4.4 contributes to smoothing the trajectory leading to directed transitions.

With this example, we have illustrated the preference-based mechanism and we have shown that it has the required properties. However, a single realization of a stochastic process is not sufficient to conclude. In the next section, we apply Kauhanen's measures complemented with a few other measures to show that the trajectory of Figure 1 is indeed representative.

\subsection{Application of Kauhanen's measures}

In order to show that the trajectory of Figure 1 is representative of the preferencebased mechanism of language change implemented through the USMwP, we apply the measures discussed in section 2.3 to the dynamical preferences scenario of section 5.1. In order to do this in a systematic manner, we vary the altered replication and the differential replication intensities by varying $q$ and $\mu$, respectively. The values of $q$ have been logarithmically varied between $10^{-5}$ and $10^{-2}$ and the values of $\mu$ have been logarithmically varied between $10^{-5}$ and $10^{-1}$. For each parameter combination, we have performed 100 runs of the model for a duration of $T=10000$ network updates (twice the duration of the time series displayed in Figure 1 ).

In order to show that the trajectory of Figure 1 is representative of the preferencebased mechanism of language change implemented through the USMwP, we apply the measures discussed in section 2.3 to the dynamical preferences scenario of section 5.1. In order to do this in a systematic manner, we vary the altered replication and the differential replication intensities by varying $q$ and $\mu$, respectively. The values of $q$ have been logarithmically varied between $10^{-5}$ and $10^{-2}$ and the values of $\mu$ have been logarithmically varied between $10^{-5}$ and $10^{-1}$. For each parameter combination, we 
have performed 100 runs of the model for a duration of $T=10000$ network updates (twice the duration of the time series displayed in Figure 1 . For each run, we have computed the following measures:

[Figure2 2here]

Results are presented in Figure 2. The 6 panels display the results for the 6 measures we considered. The vertical dashed line corresponds to the critical $q_{*} \approx 9.34 \cdot 10^{-4}$ and the green star gives the parameters used in Figure 1 . We discuss the different panels one by one below to understand the relative influence of altered and differential replications.

Let us start by discussing the dominance results. We observe that comparing with the USM (bottom of the graph, where $\mu$ is close to zero) the effect of preferences (increasing $\mu$ ) is to increase the dominance of the time series of change. This illustrates the stabilizing effect of the preference mechanism. Once a preferred variant reaches a consensus, some time is needed for the preference to decrease and for a new transition to be initiated. Looking at the position of the star, we see that the trajectory of the USMwP should be much more dominant than the corresponding USM without preferences and this is effectively what is observed in Figure 1

The next measure we consider is the monotonicity measure. We see that this measure is mainly aligned with the dominance measure and it seems that the effect of preferences on this measure is much weaker than on dominance. The critical line corresponding to $q_{*}$ is a good predictor of when the monotonicity measure almost vanishes. Looking at the star, we see that the monotonicity measure is improved by the presence of strong preferences. This is due to the smoothing effect induced by the memory encoded in the preferences. 
The smoothness measure follows the same pattern as the monotonicity and the dominance. Since the definition of this measure is related to the definition of the monotonicity measure, this is not a surprise and this measure does not provide more information than the preceding two measures.

With respect to the averaged number of shifts per 10000 time steps, in the case of the USM without preferences (bottom of the graph), we expect the graph to peak around the critical line. In fact, for small values of $q$, the stationary distribution is U-shaped, and transitions are unlikely; for large values of $q$, however, the stationary distribution is bell-shaped and the trajectory never reaches the boundaries, inhibiting transitions. So transitions should be the most frequent for intermediate values, as observed in the plot. When the differential replication is increased through the increase of $\mu$, the number of transitions augments for all values of $q$. In particular, for high values of $\mathrm{q}$, the number of transitions becomes much larger than what is observed in the USM. Looking at the position of the star, we noticed that the number of transition in an interval of length 10000 time steps is typically between 4 and 8 , which predicts between 2 and 4 transitions in the interval of 5000 time steps, which is in agreement with the two transitions observed in Figure 1 (panel d).

The measure of the length of the shifts illustrates the fact that transitions occur faster in presence of preferences. So not only does the number of transitions augments, but the transitions also shorten, which is consistent with the other measures. For instance, the increase in dominance, monotonicity and smoothness suggests that the transitions become more directed, which will be confirmed by the logisticness measure. Looking at the star, we see that the transition length is between 250 and 500 time steps, which is in agreement with Figure 1 (panel d). Before coming to the logisticness measure, we note that for high values of $q$ and low values of $\mu$, transitions become very large and 
suddenly shorten. This is an artefact originating in the finite time interval in which the shifts are measured, for instance, the maximum length of an averaged shift is around 5500 time steps, which is more than half the measured interval. This measure should continue to grow, but transitions are no longer observed because they are longer than the interval considered.

Finally, the logisticness is probably the measure for which the influence of preferences is the strongest. We observe a very clear improvement of the logisticness of trajectories when $\mu$ is increased. This is a signature that alignment of preferences is required for a successful directed transition to occur. The combination of parameters that optimise the logisticness measure corresponds to low altered replication and high differential replication, i.e. if it is difficult to innovate a new variant and new variants have a strong influence on the preferences, then fast directed changes tend to occur. But these are rare as shown by the number of shifts. This measure is quite noisy, since it can only be measured if a change did occur and, in some regions of the plot, almost no changes occurred during the simulation, leading to poor statistics. If we look at the star, we see that the logisticness value is very high even though not optimal. The time series shown in Figure 1 illustrates the S-shapedness of the changes trajectories.

Overall, the numerical simulations and the application of Kauhanen's measures demonstrates that our implementation of the preference-based mechanism has all the properties required for a well-behaved time series of language change. One can distinguish mainly four regions in Figure 2. In the lower left corner, the dynamics is dominated by a low altered replication. The stationary distribution is mainly predicted by that of the USM and a convention is usually reached and is stable, since trajectories are dominant and the number of shifts is very low. In the lower right corner, the dynamics is still dominated by altered replication, but its effect is higher and the 
stationary distribution becomes bell-shaped, losing its dominance. The number of transitions peaks around the critical value and then decreases as the stationary distribution width diminishes. In the upper left corner, differential replication is high and altered replication is low. In this region, transitions are rare, short and have a logistic shape, i.e. all the required properties of language change are realized. In the upper right corner, the altered replication process strengthens and dominance is reduced as the number of transitions augments, but these transitions are still scoring high on the logisticness measure. In this region, changes are rapid and S-shaped, this is closer to the behavior of fashions, which change frequently in a directed and S-shaped manner.

The particular example taken here and its USMwP implementation is only one possible instantiation of the preference-based mechanism. For instance, the model of Stadler et al. (2016) is also an instance of the preference-based mechanism. In our presentation, preferences are individual valuations of the variants, in Stadler's model, the individual valuation is done through the momentum of variants. The last crucial component of the mechanism is the alignment of preferences, whereas in Stadler et al. (2016), the alignment process is driven by the fact that a variant's momentum is related to temporal correlation in the variant usage and since speakers belong to the same population, their measures of momentum are correlated, which leads to alignment. We have shown that without an alignment process, S-shaped transitions are not usually observed.

\section{Discussion and conclusion}

In this paper, we have proposed a general selection mechanism to explain the shape of language change trajectories. Based on a refinement of the taxonomy of mechanisms of change of Baxter et al. (2009); ; Blythe and Croft (2012), we have shown that not all sub- 
types of RS lead to a S-shaped trajectory of change. The DSRS mechanism we propose is based on a competition between altered replication and individual differential replication. The individual valuation, or preference, for a variant is subjective and it is crucial to have a mechanism that triggers the alignment of these preferences for the preference-based selection mechanism to work. The idea to use preferences in cultural dynamics is already present in Acerbi et al. (2012) in the context of understanding fashion cycles and the importance of the alignment of the preferences, or biases, is present in Pierrehumbert et al. (2014), although not stated as such, but it can be inferred from the dynamics of the different scenarios they considered. When the biases were aligned, then informational cascades were observed, but for random biases, no such informational cascades were triggered. In Acerbi et al. (2012), the alignment process is driven by the copying dynamics typical to cultural evolution modeling. In our implementation, this alignment process is driven by a conformity bias. Our model has the advantage to also provide some insights into the actuation problem of language change. For instance, the dynamics of preferences predicts an alternation of stable and unstable periods. During stable periods, a variant is used categorically and during unstable periods, two or more variants are competing. When one variant is selected, a new trend is initiated leading to a new period of stable categorical usage. The exact dynamics of the preferences can only be approximated, since we don't really know how the variants are valued by speakers. This is a psycholinguistic problem and is outside the scope of this paper.

In section 4.3 , we have shown that our preference-based mechanism is related to the replicator-mutation dynamics and that the preferences can be interpreted as subjective fitnesses of variants in the linguistic ecosystem of an agent and that the mutation process affecting this ecosystem is driven by contact between speakers, which can also 
be construed as migration between speakers' linguistic ecosystems. This biological analogy is interesting and can lead to the development of our understanding of the linguistic system self-organisation using techniques from ecology. We believe that this is an idea worth exploring and that it could lead to an interesting extension of this work.

Using Kauhanen's measures complemented with a logisticness measure, we have demonstrated that the preference-based mechanism indeed reproduces all the required properties of a well-behaved trajectory of language change. It would be interesting to apply these measures to other models, such as Stadler et al. (2016) momentum-based model to compare the results. This is outside the scope of this paper, but could be an interesting future direction of research. The advantage of using measures of the time series is that it provides information about the typical trajectories predicted by a theory and one could apply these measures to actual time series of change to enable a quantitative comparison between language change models, opening a new field of investigation of this complex process.

To conclude, we link our results to changes in linguistic features. As mentioned in Section 2.1. intensifiers tend to change much like fashions, i.e. relatively frequently. According to our analysis, intensifiers are characterized by a fairly high altered replicated as well as a rather high differential replication. Therefore, they should be placed in the upper right corner of Figure 2 which predicts relatively frequent directed changes, as expected. For more stable linguistic variables, such as word order or negation patterns, the altered replication process can be assumed to be much weaker. If we assume a similar differential replication, this type of change should be placed in the upper left corner of Figure 2, which predict rare, directed and S-shaped transitions, as expected. Therefore, the preference-based mechanism presented in this paper is able to explain 
both rare and more frequent changes and predicts directed transitions that are mainly S-shaped.

\section{References}

Acerbi, Alberto, Stefano Ghirlanda, and Magnus Enquist. 2012. The logic of fashion cycles. PloS one 7(3): e32,541.

Asch, S.E. 1956. Studies of independence and conformity: I. a minority of one against a unanimous majority. Psychological Monographs: General and Applied 70(9): 1-70.

Bailey, Charles-James N. 1973. Variation and linguistic theory. .

Baxter, Gareth and William Croft. 2016. Modeling language change across the lifespan: Individual trajectories in community change. Language Variation and Change 28(02): $129-173$.

Baxter, Gareth J, Richard A Blythe, William Croft, and Alan J McKane. 2006. Utterance selection model of language change. Physical Review E 73(4): 046,118.

- 2009. Modeling language change: An evaluation of trudgill's theory of the emergence of new zealand english. Language Variation and Change 21(02): 257-296.

Beckner, Clay, Richard A. Blythe, Joan Bybee, Morten H. Christiansen, William Croft, Nick C. Ellis, John Holland, Jinyun Ke, Diane Larsen-Freeman, and Tom Schoenemann. 2009. Language is a complex adaptibe system: Position paper. Language Learning 59: Suppl. 1, 1-26.

Beuls, Katrien, Luc Steels, and Sebastian Höfer. 2012. The emergence of internal agreement systems. Experiments in Cultural Language Evolution. John Benjamins, Amsterdam .

Beuls, Katrien, Luc Steels et al. 2013. Agent-based models of strategies for the emergence and evolution of grammatical agreement. PloS one 8(3): e58,960. 
Blythe, Richard A. and William Croft. 2012. S-curves and the mechanisms of propagation in language change. Language 88(2): 269-304.

Bybee, Joan. 2001. Phonology and language use, vol. 94. Cambridge University Press.

. 2007. Frequency of use and the organization of language. Oxford University Press.

_. 2010. Language, usage and cognition. Cambridge University Press.

Cedergren, Henrietta. 1987. The spread of language change: Verifying inferences of linguistic diffusion. Language spread and language policy: Issues, implications, and case studies $45-60$.

Chambers, Jack K, Peter Trudgill, and Natalie Schilling. 2002. The handbook of language variation and change, vol. 129. Blackwell.

Cooper, Russell. 1998. Coordination games. Cambridge University Press.

Croft, William. 2000. Explaining language change: An evolutionary approach. Pearson Education.

- 2008. Evolutionary linguistics. Annual Review of Anthropology 37(1): 219.

De Clerck, Bernard and Timothy Colleman. 2013. From noun to intensifier: massa and massa's in flemish varieties of dutch. Language Sciences 36: 147-160.

de Saussure, Ferdinand. 1959. Course in general linguistics. New York: The Philosophical Library, Inc.

Denison, David. 2003. Log (ist) ic and simplistic s-curves. Motives for language change 54: 70.

Fedzechkina, Maryia. 2014. Communicative efficiency, language learning, and language universals. Ph.D. thesis, University of Rochester.

Fedzechkina, Maryia, Becky Chu, T. Florian Jaeger, and John Trueswell. 2016. Processing preferences shape language change. In S.G. Roberts, C. Cuskley, L. McCrohon, L. Barceló-Coblijn, O. Fehér, and T. Verhoef (eds.) The Evolution of Lan- 
guage: Proceedings of the 11th International Conference (EVOLANGX11). Online at http://evolang.org/neworleans/papers/101.html.

Finley, Sara and William Badecker. 2007. Towards a substantively biased theory of learning. In Berkeley Linguistics Society, vol. 33, 142-154.

Ghanbarnejad, Fakhteh, Martin Gerlach, José M Miotto, and Eduardo G Altmann. 2014.

Extracting information from S-Curves of language change. Journal of The Royal Society Interface 11(101): 20141,044.

Giles, Howard. 1973. Accent mobility: A model and some data. Anthropological linguistics 87-105.

Giles, Howard and Peter Powesland. 1997. Accommodation theory. In Sociolinguistics, 232-239. Springer.

Giles, Howard and Philip Smith. 1979. Accommodation theory: Optimal levels of convergence, 45-65. Baltimore: University Park Press.

Greenberg, Joseph H. 1960. A quantitative approach to the morphological typology of language. International journal of American linguistics 26(3): 178-194.

Halliday, Michael Alexander Kirkwood. 1978. Language as social semiotic. London Arnold.

Haspelmath, Martin. 2004. On directionality in language change with particular reference to grammaticalization. Typological Studies in Language 59: 17-44.

Hofbauer, Josef. 1985. The selection mutation equation. Journal of mathematical biology 23(1): 41-53.

Hull, David L. 1980. Individuality and selection. Annual review of ecology and systematics 11(1): 311-332. 2001. Science and selection. Cambridge Un. Press, Cambridge . 
2010. Science as a process: an evolutionary account of the social and conceptual development of science. University of Chicago Press.

Ito, Rika and Sali A Tagliamonte. 2003. Well weird, right dodgy, very strange, really cool: Layering and recycling in english intensifiers. Language in Society 32(02): 257-279.

Jansson, Fredrik, Mikael Parkvall, and Pontus Strimling. 2015. Modeling the evolution of creoles. Language Dynamics and Change 5(1): 1-51.

Kauhanen, Henri. 2016. Neutral change. Journal of Linguistics 1-32.

Kirby, Simon, Monica Tamariz, Hannah Cornish, and Kenny Smith. 2015. Compression and communication in the cultural evolution of linguistic structure. Cognition 141: $87-102$.

Komarova, Natalia L. 2004. Replicator-mutator equation, universality property and population dynamics of learning. Journal of Theoretical Biology 230(2): 227-239.

Kroch, Anthony S. 1989. Reflexes of grammar in patterns of language change. Language variation and change 1(03): 199-244.

Labov, William. 2001. Principles of linguistic change Volume 2: Social factors, vol. 29. Blackwell.

2011. Principles of linguistic change, cognitive and cultural factors, vol. 3. John Wiley \& Sons.

Le Page, Robert Brock and Andrée Tabouret-Keller. 1985. Acts of identity: Creole-based approaches to language and ethnicity. CUP Archive.

Lewis, Hannah M and Kevin N Laland. 2012. Transmission fidelity is the key to the build-up of cumulative culture. Philosophical Transactions of the Royal Society of London B: Biological Sciences 367(1599): 2171-2180.

MacWhinney, Brian, Andrej Malchukov, and Edith Moravscik. 2014. Competing motivations in grammar and usage. OUP Oxford. 
Michaud, J. 2014. On the emergence of bilingualism in a communication "ALL" task as a result of competition between social conformism and language simplification. In E. A. Cartmill, S. Roberts, H. Lyn, and H. Cornish (eds.) The evolution of language: Proceedings of the 10th International conference (EVOLANGX), 185-192. World Scientific.

Michaud, Jérôme. 2017. Continuous time limits of the utterance selection model. Phys. Rev. E 95: 022,308. doi:10.1103/PhysRevE.95.022308. URL http:// link . aps . org/ doi/10.1103/PhysRevE.95.022308.

Milroy, James and Lesley Milroy. 1985. Linguistic change, social network and speaker innovation. Journal of linguistics 21(02): 339-384.

Milroy, Lesley. 1980. Language and social networks. Blackwell Oxford.

Mitchener, W Garrett. 2009. A stochastic model of language change through social structure and prediction-driven instability. language acquisition 24: 25.

Mühlenbernd, Roland and Jérôme Michaud. 2017. The utterance selection model and different types of replicator selection. In 23 rd International Conference on Historical Linguistics.

Nedergaard Thomsen, Ole. 2006. Competing models of linguistic change: Evolution and beyond, vol. 279. John Benjamins Publishing.

Nevalainen, Terttu, Helena Raumolin-Brunberg, and Heikki Mannila. 2011. The diffusion of language change in real time: Progressive and conservative individuals and the time depth of change. Language Variation and Change 23(01): 1-43.

Newmeyer, Frederick J. 1998. Language form and language function. MIT press.

Partington, Alan. 1993. Corpus evidence of language change. Text and Technology. In Honour of John Sinclair 177-192.

Pierrehumbert, Janet B, Forrest Stonedahl, and Robert Daland. 2014. A model of grassroots changes in linguistic systems. arXiv preprint arXiv:1408.1985 . 
Reali, Florencia and Thomas L Griffiths. 2009. The evolution of frequency distributions: Relating regularization to inductive biases through iterated learning. Cognition 111(3): $317-328$.

Rogers, Everett M. 2010. Diffusion of innovations. Simon and Schuster.

Roth, Alvin E and Ido Erev. 1995. Learning in extensive-form games: Experimental data and simple dynamic models in the intermediate term. Games and economic behavior 8(1): 164-212.

Smith, A.D.M., B. Skarebela, and M. Tamariz. 2010. Exploring the nature of a systematicity bias: an experimental study. In A.D.M. Smith, M. Schouwstra, B. de Boer, and K. Smith (eds.) The Evolution of Language: Proceedings of the 8th International Conference (EVOLANG8), 289-296. World Scientific.

Stadler, Kevin. 2016. Direction and directedness in language change: An evolutrionary model of selection by trend-amplification. Ph.D. thesis, University of Edinburgh.

Stadler, Kevin, Richard A Blythe, Kenny Smith, and Simon Kirby. 2016. Momentum in language change. Language Dynamics and Change 6(2): 171-198.

Steels, Luc. 2007. The recruitment theory of language origins. In Emergence of communication and language, 129-150. Springer.

Steels, Luc and Martin Loetzsch. 2012. The grounded naming game. Experiments in cultural language evolution 3: 41-59.

Sutton, Richard S and Andrew G Barto. 1998. Reinforcement learning: An introduction, vol. 1. MIT press Cambridge.

Tadelis, Steven. 2013. Game theory: an introduction. Princeton University Press.

Tagliamonte, Sali A. 2008. So different and pretty cool! recycling intensifiers in toronto, canada. English Language and Linguistics 12(02): 361-394. 
. 2012. Variationist sociolinguistics: Change, observation, interpretation, vol. 40. John Wiley \& Sons.

. 2015. Making Waves: The Story of Variationist Sociolinguistics. John Wiley \& Sons.

Weinreich, U., W. Labov, and M. I. Herzog. 1968. Empirical foundations for a theory of language change. In W. P. Lehmann and Y. Malkiel (eds.) Directions for historical linguistics, 95-195. Austin: University of Texas Press. 


\section{A Mathematical details}

In this appendix, we provide some mathematical details on the derivation of the mathematical formulations of the model.

\section{A.1 Accommodation rule with 2 variants}

\section{Proposition 1}

The learning rule given in Equation (6) can be rewritten as Equation (7) for $\delta x_{v}^{(i), n}$.

\section{Proof}

We start from the learning rule (6), we have

$$
\begin{aligned}
x_{1}^{(i), n+1} & =x_{1}^{(i), n}\left(1-\alpha_{1}^{(i), n}\right)\left(1-u_{1}^{(j), n}\right)+\left(x_{1}^{(i), n}+\alpha_{2}^{(i), n} x_{2}^{(i), n}\right) u_{1}^{(j), n} \\
& =x_{1}^{(i), n}-\alpha_{1}^{(i), n} x_{1}^{(i), n}+\alpha_{1}^{(i), n} x_{1}^{(i), n} u_{1}^{(j), n}+\alpha_{2}^{(i), n} x_{2}^{(i), n} u_{1}^{(j), n} \\
& =x_{1}^{(i), n}+u_{1}^{(j), n}\left(\alpha_{1}^{(i), n} x_{1}^{(i), n}+\alpha_{2}^{(i), n} x_{2}^{(i), n}\right)-\alpha_{1}^{(i), n} x_{1}^{(i), n},
\end{aligned}
$$

which implies that

$$
\delta x_{1}^{(i), n}=u_{1}^{(j), n} \sum_{w=1}^{2} \alpha_{w}^{(i), n} x_{w}^{(i), n}-\alpha_{1}^{(i), n} x_{1}^{(i), n}
$$

\section{Proposition 2}

The learning rule given in Equation (7) has the following properties:

1. $x_{v}^{(i), n} \in[0,1], \forall n$, if $x_{v}^{(i), 0} \in[0,1]$;

2. the learning rule conserves probability, i.e. $\sum_{w} \delta x_{w}^{(i), n}=0$.

\section{Proof}


1. To prove this point, one needs to prove that $-x_{v}^{(i), n} \leq \delta x_{1}^{(i), n} \leq 1-x_{v}^{(i), n}$.

We prove the two inequalities independently:

(a) $\quad-x_{v}^{(i), n} \leq \delta x_{1}^{(i), n}:$ In order to show this point, note that $0 \leq u_{v}^{(i), n}, \alpha_{v}^{(i), n} \leq 1$, which implies that

$$
\delta x_{1}^{(i), n} \geq-\alpha_{1}^{(i), n} x_{1}^{(i), n} \geq-x_{1}^{(i), n} .
$$

(b) $\quad \delta x_{1}^{(i), n} \leq 1-x_{v}^{(i), n}$ : Extracting the terms that depends on $x_{1}^{(i), n}$, we

get

$$
\delta x_{1}^{(i), n}=\left(u_{1}^{(j), n}-1\right) \alpha_{1}^{(i), n} x_{1}^{(i), n}+\sum_{w \neq 1}^{2} \alpha_{w}^{(i), n} x_{w}^{(i), n} .
$$

The first term is negative, since $u_{1}^{(j), n} \leq 1$. We then obtain

$$
\delta x_{1}^{(i), n} \leq \sum_{w \neq 1}^{2} \alpha_{w}^{(i), n} x_{w}^{(i), n} \leq \sum_{w \neq 1}^{2} x_{w}^{(i), n}=1-x_{1}^{(i), n} .
$$

2. To prove this point, we simply sum over the different jumps. We obtain

$$
\begin{aligned}
\sum_{w=1}^{2} \delta x_{w}^{(i), n} & =\sum_{w=1}^{2}\left(u_{w}^{(j), n} \sum_{w^{\prime}=1}^{2} \alpha_{w^{\prime}}^{(i), n} x_{w^{\prime}}^{(i), n}-\alpha_{w}^{(i), n} x_{w}^{(i), n}\right) \\
& =\sum_{w^{\prime}=1}^{2} \alpha_{w^{\prime}}^{(i), n} x_{w^{\prime}}^{(i), n} \underbrace{\sum_{w=1}^{2} u_{w}^{(j), n}}_{=1}-\sum_{w=1}^{2} \alpha_{w}^{(i), n} x_{w}^{(i), n}=0 .
\end{aligned}
$$

\section{A.2 Generalization to $V$ variants}

In this section, we show that the learning rule (8) for a arbitrary number of variants $V$.

Using the same reasoning that leads to Equation (6), we obtain

$$
x_{v}^{(i), n+1}=x_{v}^{(i), n}\left(1-\alpha_{v}^{(i), n}\right)\left(1-u_{v}^{(j), n}\right)+\left(x_{v}^{(i), n}+\sum_{w \neq v} \alpha_{w}^{(i), n} x_{w}^{(i), n}\right) u_{v}^{(j), n},
$$


where the same interpretation of the terms applies: penalization occurs whenever agent $j$ chooses a variant different from variant $v$ and in the case when she chooses variant $v$, then the variant get reinforced by the sum of probability weights originating in the penalization of the other variants.

\section{Proposition 3}

The learning rule given in Equation (A.22) can be rewritten as Equation (8) for $\delta x_{v}^{(i), n}$.

\section{Proof}

We start from the learning rule A.22, we have

$$
\begin{aligned}
x_{1}^{(i), n+1} & =x_{1}^{(i), n}\left(1-\alpha_{1}^{(i), n}\right)\left(1-u_{1}^{(j), n}\right)+\left(x_{v}^{(i), n}+\sum_{w \neq v} \alpha_{w}^{(i), n} x_{w}^{(i), n}\right) u_{1}^{(j), n} \\
& =x_{1}^{(i), n}-\alpha_{1}^{(i), n} x_{1}^{(i), n}+u_{1}^{(j), n} \alpha_{1}^{(i), n} x_{1}^{(i), n}+u_{1}^{(j), n} \sum_{w \neq v} \alpha_{w}^{(i), n} x_{w}^{(i), n} \\
& =x_{1}^{(i), n}+u_{1}^{(j), n} \sum_{w} \alpha_{w}^{(i), n} x_{w}^{(i), n}-\alpha_{1}^{(i), n} x_{1}^{(i), n},
\end{aligned}
$$

which implies that

$$
\delta x_{1}^{(i), n}=u_{1}^{(j), n} \sum_{w=1}^{V} \alpha_{w}^{(i), n} x_{w}^{(i), n}-\alpha_{1}^{(i), n} x_{1}^{(i), n} .
$$

The generalized learning rule has the same properties than the learning rule for two variants and Proposition 2 still applies, since it doesn't depend on how many variants are present.

\section{A.3 Connection with Replicator-Mutator dynamics}

In this section, we show the correspondence between Equation (12) and the ReplicatorMutator dynamics given by

$$
\dot{x}_{v}=\sum_{w} x_{w} f_{w}(\boldsymbol{x}) Q_{w v}-\phi(\boldsymbol{x}) x_{v}, \quad \phi(\boldsymbol{x})=\sum_{w} x_{w} f_{w}(\boldsymbol{x}),
$$




\section{Proposition 4}

Eq. (12) can be written as a replicator-mutator equation with the definitions

$$
\begin{aligned}
f_{v}^{(i)}(\boldsymbol{x}) & :=-\alpha_{v}^{(i)}, \\
Q_{w v}^{(i j)} & :=x_{v}^{(i)}-u_{v}^{(j)}+\delta_{v w} .
\end{aligned}
$$

\section{Proof}

$$
\begin{aligned}
\dot{x}_{v}^{(i)} & =u_{v}^{(j)} \sum_{w} \alpha_{w}^{(i)} x_{w}^{(i)}-\alpha_{v}^{(i)} x_{v}^{(i)} \\
& =u_{v}^{(j)} \sum_{w} \alpha_{w}^{(i)} x_{w}^{(i)}-\alpha_{v}^{(i)} x_{v}^{(i)}-x_{v}^{(i)} \sum_{w} \alpha_{w}^{(i)} x_{w}^{(i)}+x_{v}^{(i)} \sum_{w} \alpha_{w}^{(i)} x_{w}^{(i)} \\
& =\sum_{w} \alpha_{w}^{(i)} x_{w}^{(i)} u_{v}^{(j)}-\sum_{w} \alpha_{w}^{(i)} x_{w}^{(i)} \delta_{v w}-x_{v}^{(i)} \sum_{w} \alpha_{w}^{(i)} x_{w}^{(i)}+x_{v}^{(i)} \sum_{w} \alpha_{w}^{(i)} x_{w}^{(i)} \\
& =\sum_{w} x_{w}^{(i)} \underbrace{\left(-\alpha_{w}^{(i)}\right)}_{=: f_{w}^{(i)}(\boldsymbol{x})} \underbrace{\left(x_{v}^{(i)}-u_{v}^{(j)}+\delta_{v w}\right)}_{=: Q_{w v}^{(i j)}}-x_{v}^{(i)} \sum_{w} x_{w}^{(i)} \underbrace{\left(-\alpha_{w}^{(i)}\right)}_{=: f_{w}^{(i)}(\boldsymbol{x})},
\end{aligned}
$$

which concludes the proof. 
Table 1

Interpretation of the parameters of the USM

\begin{tabular}{cll}
\hline \hline Parameter & Name & Interpretation \\
\hline$N$ & Number of agents & Population size \\
$V$ & Number of variants & $\begin{array}{l}\text { Sociolinguistic variable } \\
\text { Innovations and intra-linguistic dynamics, } \\
\text { altered or differential replication }\end{array}$ \\
& Mutation matrix & Variability of speech \\
$L$ & Length of utterances & WIS \\
$h^{(i j)}$ & Attention parameter & NIS and network structure \\
$G^{(i j)}$ & Probability of interaction & Importance of recent utterances \\
$\lambda$ & Learning rate & $\begin{array}{l}\text { Perception biases and SORS or DSRS, can } \\
\text { also be used to model DORS or SSRS. }\end{array}$ \\
\hline$f(\cdot)$ & Perception function & \\
\hline \hline
\end{tabular}



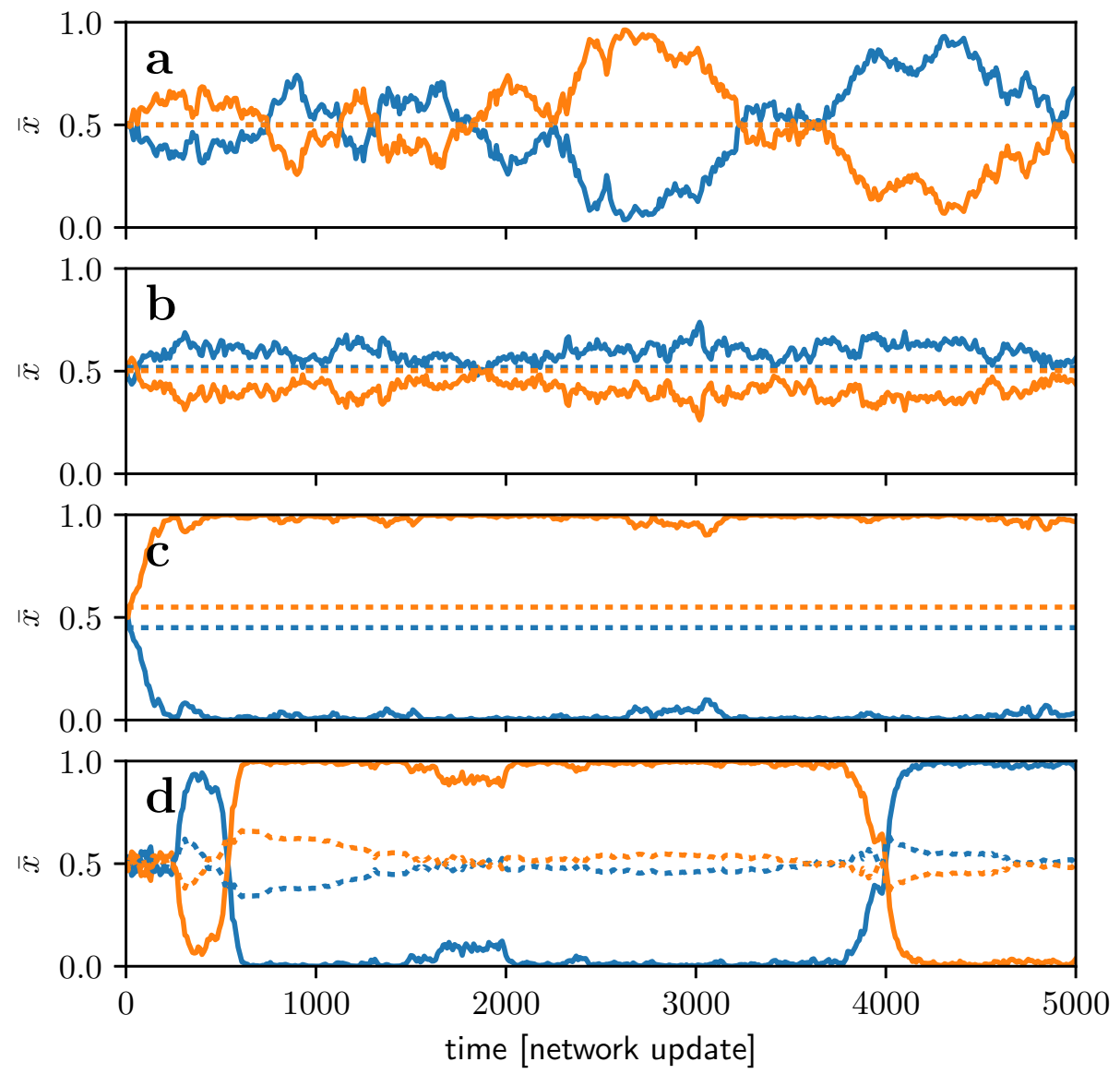

Figure 1

Illustration of the time series of the USM with preferences. Continuous lines are time series of variant usage and dashed lines display the corresponding preferences. Panel a displays a typical trajectory of the USM where the preferences are fixed to 0.5, which is an example of NE selection mechanism. Panel $\mathbf{b}$ displays a typical trajectory of the USM where the preferences are randomly set, which is an example of a SSRS mechanism. Panel $\mathbf{c}$ displays a typical trajectory of the USM where the preferences are fixed to 0.55 for one variant and 0.45 for the other, which is an example of an SORS mechanism. Panel $\mathbf{d}$ displays a typical trajectory of the USM where the preferences are evolved, which is an example of the DSRS mechanism. The simulations are performed on a random regular network of degree $k=3$ with $N=20$ agents, where all the edges are sample uniformly and where $h^{(i j)}=0.5$ for all agents. The linguistic variable has $V=2$ variants and utterance are of length $L=2$. The innovation/error matrix $M$ is controlled by the parameter $q=0.001$. The learning rule is set to $\lambda=0.1$ and the parameter controlling the preference update in panel $\mathbf{d}$ is set to $\mu=0.05$. The time axis is measured in network updates, i.e. for the time to be incremented by one, all the edges of the network are updating and all agents interact as many times as their degree. 

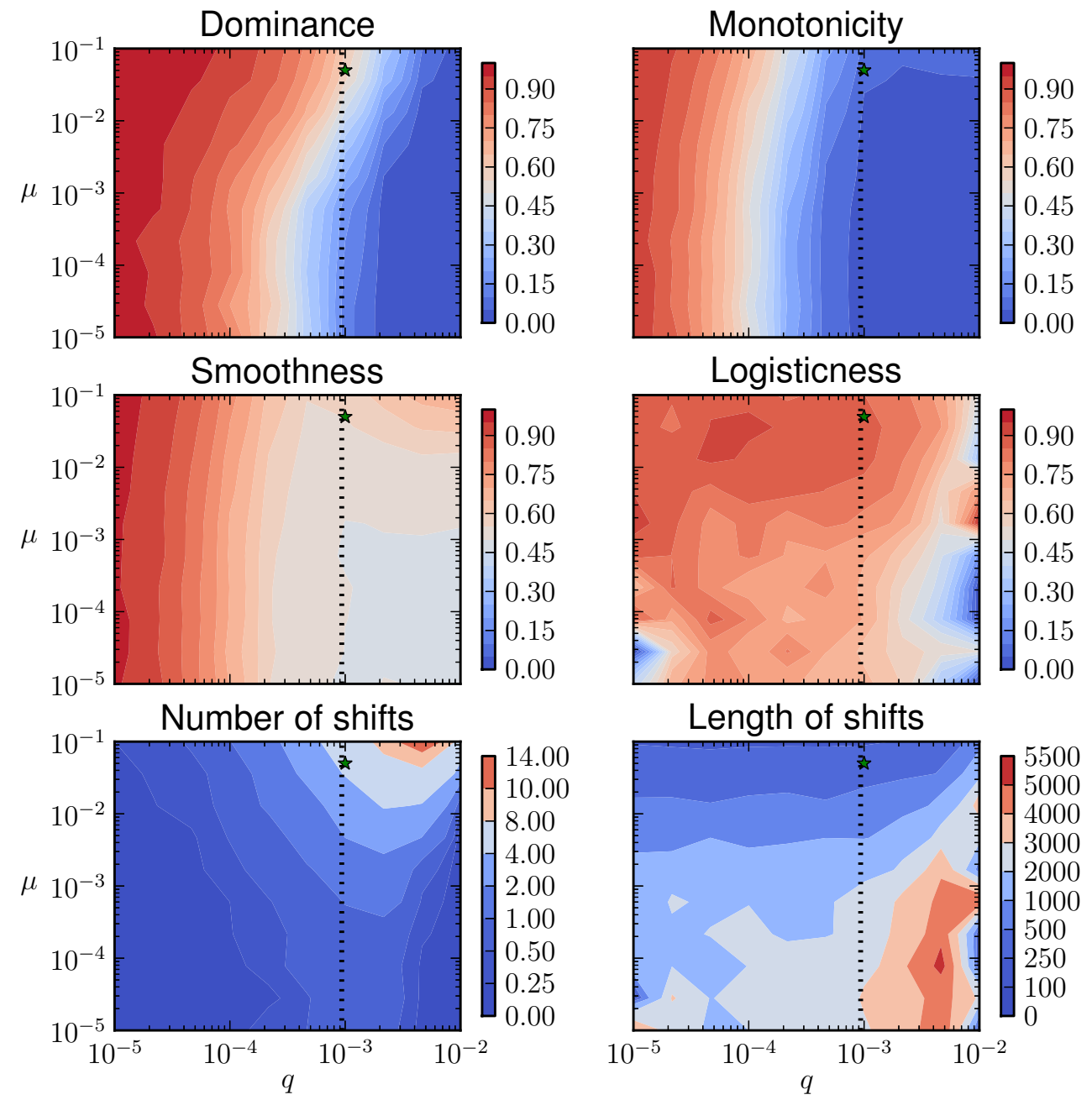

\section{Figure 2}

Results for the application of time series measures. For each measures, 10 logarithmically spaced values for $q$ and $\mu$ have been selected and, for each parameter combination, 100 runs of length 10000 network updates have been performed. The different panels display the averaged measures obtained. The dashed vertical line corresponds to the critical $q_{*}$ value of the USM with constant preferences $\alpha=0.5$ for all agents and for all variants. The green star illustrates the parameter combination used in Figure 1 The original Kauhanen's measures are displayed in the Dominance, Monotonicity and Number of shifts panels. We added the Smoothness, the Logisticness and the Length of shifts measures. 
